\title{
The diagnostic value of provocative clinical tests in ulnar neuropathy at the elbow is marginal
}

\section{Authors:}

R Beekman, MD, PhD (1, 2), AHCML Schreuder, MD (1), CAM Rozeman, MD, PhD (2), PJ Koehler, MD, PhD (1), BMJ Uitdehaag, MD, PhD (3)

Departments of Neurology (1) and Neurophysiology (2), Atrium Medical Centre, Heerlen, The Netherlands. Department of Epidemiology and Biostatistics (3), VU University Medical Centre, Amsterdam, The Netherlands

\section{Correspondence:}

Roy Beekman, MD, PhD, Department of Neurology, Atrium Medical Centre, PO Box 4446, 6401 CX, Heerlen, The Netherlands, Phone 31.45.5766702, Fax 31.45.5767416, e-mail:

r.beekman@atriummc.nl

Keywords: ulnar neuropathy, physical examination, ultrasonography 


\section{ABSTRACT}

Background: Provocative clinical tests are often performed in the diagnosis of ulnar neuropathy at the elbow (UNE) although the evidence for the usefulness of these tests is limited. The aim of this study was to determine the diagnostic value of provocative clinical tests in the diagnosis of UNE in a relevant spectrum of patients and controls.

Methods: We performed a prospective cohort study in consecutive patients clinically suspected for UNE. All patients underwent a neurological exam and four commonly used provocative clinical tests (Tinel's test, flexion compression test, palpating for local ulnar nerve tenderness and nerve thickening). Subsequently in all patients a reference standard test comprising of electrophysiological studies and neurosonography was independently assessed.

Results: 192 eligible patients completed the study protocol. UNE was diagnosed in 137 and an alternative diagnosis was made in 55 patients. The sensitivity, specificity, PPV and NPV were: Tinel's test 62, 53, 77 and 30\%, flexion compression test 61, 40, 72 and $29 \%$, palpating for nerve thickening $28,87,84$ and $33 \%$, palpating for nerve tenderness 32, 80, 80 and 32\%. Logistic regression and ROC curves showed that the added value of one or more provocative tests over routine clinical examination is minimal.

Conclusion: The diagnostic value of provocative clinical tests in UNE is poor. 


\section{INTRODUCTION}

Ulnar neuropathy at the elbow (UNE) is clinically easily suspected. However, it is often not possible to definitely localize the lesion at the elbow or differentiate from other disorders (e.g., ulnar neuropathy at the wrist, lower brachial plexopathy, or C8 radiculopathy) on the basis of clinical examination of sensory and motor functions. Sensory deficits in the area of the cutaneous dorsal ulnar nerve or weakness of the flexor carpi ulnaris and flexor digitorum profundus muscles localize the lesion above the wrist but these functions are often normal.(1) A final diagnosis is made after electrophysiological or sonographic examination of the ulnar nerve.(2)

Provocative clinical tests are often applied and recommended to localize the lesion and make the diagnosis in UNE. Palpating for nerve thickening and local tenderness, Tinel's test, and flexion compression tests have all been advocated.(3) However, there are only few studies to substantiate these recommendations and moreover, they all have serious methodological deficits. In patients with carpal tunnel syndrome the efficacy of provocative tests was low.(4)

The aim of this study was to determine the diagnostic value of provocative clinical tests (Tinel's test, flexion compression test, palpating for local ulnar nerve tenderness and nerve thickening) in patients in whom a diagnosis of UNE was considered. 


\section{METHODS}

Between December 2006 and December 2008 we prospectively studied the usefulness of provocative clinical testing in patients referred to the outpatient department of neurology of Atrium Medical Centre, a large general teaching hospital. All patients in whom UNE was considered in the differential diagnosis after initial history taking were eligible for the study. Symptoms consistent with an ulnar neuropathy were defined as numbness and paraesthesias of the fourth and fifth digits of the hand, weakness or clumsiness of the muscles innervated by the ulnar nerve. In patients with bilateral complaints, only the most severely affected side according to the patient was used in all analyses. The exclusion criteria were acute traumatic origin, history of a polyneuropathy or genetically proven hereditary neuropathy with liability to pressure palsies, and findings consistent with polyneuropathy at physical examination. Patients were first clinically examined by one of two experienced neurologists (index test) and then referred for electrophysiological and sonographic studies (reference test) as described below. No ethical approval or informed consent was required because all tests were performed as part of standard patient care.

\section{Index test - clinical examination}

The index test consisted of a routine neurological exam and provocative tests. During the routine neurological exam motor function, sensory function, tendon reflexes, coordination, and cranial nerves were tested. In every case we at least recorded pinprick sensation in the area of the ulnar superficial terminal, palmar cutaneous, and dorsal cutaneous sensory branches and strength of the first dorsal interosseous (FDI), abductor digiti minimi (ADM), flexor carpi ulnaris (FCU), and flexor digitorum profundus of digits four and five (FDP) muscles using the Medical Research Council Rating Scale. Subsequently the following tests were performed in each patient in random order:

(1) Tinel's test: tapping lightly at the ulnar nerve around the medial epicondylar groove; the test is positive if the patient reports tingling or electrical sensations radiating to the fourth and fifth digits. $(3,7)$

(2) Flexion compression test: the examiner keeps the patient's elbow maximally flexed with the wrist in neutral position during 60 seconds while giving compression with index and middle finger just proximal to the cubital tunnel; the test is positive when the patient reports tingling sensations in the ulnar sensory area within 60 seconds. (7)

(3) Palpating for local nerve tenderness and thickening: the examiner palpates the ulnar nerve around the elbow, decides whether the ulnar nerve is thickened and asks the patient if there is tenderness in that area.

After these tests the examiner made a differential diagnosis and indicated the likelihood of UNE in each patient (probable, possible, unlikely). Subsequently, all patients were referred for ulnar electrophysiological and sonographic studies as described below (reference test). Additional electrophysiological studies and imaging studies (e.g., of the cervical spine or brachial plexus) were ordered if a possible radiculopathy, brachial plexopathy, or other neuropathy was also considered in the differential diagnosis. Patients in whom (after ancillary tests) a diagnosis other than UNE was made were analysed as patient controls. In case a patient had UNE and another arm condition that patient was analysed as UNE patient.

\section{Reference test - electrophysiological studies / sonography}

The standard criterion for UNE consisted of one or more symptoms or signs of a possible ulnar neuropathy and one or two of the following: electrophysiological evidence of an ulnar neuropathy at the elbow or ulnar nerve thickening demonstrated during sonography. The electrophysiological and sonographic exams were carried out by different examiners who were unaware of each other's test 
results. Both examiners were unaware of the clinical findings of the neurologist performing the clinical examination including the provocative tests.

\section{Electrophysiological studies}

Ulnar sensory and motor nerve conduction studies were performed with the elbow flexed at $90^{\circ}$. If necessary, skin temperature was raised to $>32^{\circ} \mathrm{C}$ using hot water baths. Surface stimulation was performed with the cathode placed at the proximal wrist crease, $4 \mathrm{~cm}$ distal to the medial epicondyle and 4 to $6 \mathrm{~cm}$ above the elbow (range of the across-elbow distance was 8 to $10 \mathrm{~cm}$ ). Compound muscle action potentials (CMAP) were recorded from the ADM and FDI muscles using surface electrodes in a belly-tendon montage. The most severe conduction abnormalities at the elbow in one of these two derivations were used for analysis. Sensory nerve action potentials (SNAP) were obtained antidromically using ring electrodes placed over the fifth digit.

In accordance with guidelines of the Netherlands Society of Clinical Neurophysiology and AAEM, ulnar neuropathy was localized at the elbow if one or more of the following abnormalities were found (reference values derived from our previous studies, mean $\pm 2 \mathrm{SD}$ ): reduction of the CMAP from the below- to the above-elbow site of $>16 \%$ (block), motor nerve conduction velocity (MNCV) across the elbow of $<46 \mathrm{~m} / \mathrm{s}$ (slowing), MNCV at the across-elbow segment $>15 \mathrm{~m} / \mathrm{s}$ slower than at the forearm segment (differential slowing). Cut-off values for an abnormally low action potential were (mean -2 $\mathrm{SD}$ ): $<5.5 \mathrm{mV}$ for the ADM CMAP, $<7.0 \mathrm{mV}$ for the FDI CMAP, and $<6.5 \mu \mathrm{V}$ for the digit V ulnar SNAP.(2)

\section{Sonography}

Using a 5- to $10-\mathrm{MHz}$ linear-array transducer the ulnar nerve at the elbow was visualized on longitudinal scans measuring the diameter of the ulnar nerve within the echogenic rim surrounding the nerve accurate to $0.1 \mathrm{~mm}$. This measurement was done at the level of the medial epicondyle and repeated $2 \mathrm{~cm}$ proximal to and $2 \mathrm{~cm}$ distal to this level. Sonography was considered abnormal if the diameter was increased at any of these levels, the cut-off values being respectively $2.6 \mathrm{~mm}, 2.5 \mathrm{~mm}$, and $2.7 \mathrm{~mm}$.(2) In a previous study we foud a sensitivity of $81 \%$ and specificity of $91 \%$ for sonography in the diagnosis of UNE.(2) Figure 1 shows an ultrasound image of an enlarged ulnar nerve.

\section{STATISTICAL ANALYSIS}

All continuous and normally distributed variables were analysed using the Student t-test, discontinuous and nonnormally distributed variables using the Mann-Whitney test. The chi-square test was used to compare proportions of independent variables. In all analyses a p-value $<0.05$ was considered to be significant.

To determine the performance of the provocative tests, each test result was categorized as true positive (TP), false negative (FN), true negative (TN), and false positive (FP) according to the reference test. The following predictive characteristics were calculated: sensitivity (TP/[TP + FN]), specificity $(\mathrm{TN} /[\mathrm{TN}+\mathrm{FP}])$, accuracy $([\mathrm{TP}+\mathrm{TN}] /[\mathrm{TP}+\mathrm{TN}+\mathrm{FP}+\mathrm{FN}])$, positive predictive value $(\mathrm{PPV})(\mathrm{TP} /[\mathrm{TP}+$ 
FP]), and negative predictive value (NPV) (TN/[TN + FN]). Furthermore, we determined likelihood ratios (LRs). In this study the LR is the ratio of the probability of the specific test result in patients with UNE to the probability in patients without UNE. We performed the following calculations: LR of a positive test $(L R+)=$ sensitivity/(1 - specificity), LR of a negative test $(L R-)=(1$ - sensitivity $) /$ specificity.

Multiple logistic regression was used to further evaluate the value of the tests. In order to approach the clinical diagnostic process, the selected variables were grouped into three subgroups: 1) age, gender and most affected side; 2) presence of motor function and sensory function disturbances related to the ulnar nerve; 3 ) results of provocative tests. Successively, three logistic regression analyses were done in which the variables of the three groups were entered, starting with subgroup 1, followed by subgroups 1 and 2, then finally subgroups 1 to 3 . Thus, three successive models were constructed. The three final models were compared using the likelihood ratio test. Receiver operating characteristic (ROC) curves were constructed for each model and the areas under the curve (AUCs) and 95\% confidence intervals $(95 \% \mathrm{Cl})$ were estimated. Moreover a logistic regression analysis (Backward Wald method, entry 0.10 , removal 0.20 ) was performed including all predictor variables.

ROC curves were also constructed and AUC estimated for the number of positive provocative tests. Finally, the diagnostic value was graphically expressed by plotting the post-test probability as a function of the pre-test probability for negative and positive test results.

All statistical analyses were performed using SPSS (version 15.0; SPSS Inc., Chicago, III). 


\section{RESULTS}

\section{Patients and controls}

The flow diagram of the study is shown in figure 2. In 192 patients UNE was considered in the differential diagnosis after history taking. All these patients underwent the index and reference tests according to the protocol. Ultimately 137 patients had UNE while 55 had an alternative diagnosis. These other diagnoses were: cervical radiculopathy $(n=8)$, carpal tunnel syndrome $(n=10)$, stroke involving the precentral motor cortex of the hand $(n=2)$, brain metastasis $(n=1)$, cervical myelopathy $(n=1)$, epicondylitis or aspecific arm pain $(n=33)$.

Of the UNE patients, 79 had ulnar sensory and motor signs, 31 ulnar sensory signs only, and 27 a normal neurological exam. The clinical characteristics of patients and controls are described in table 1.

Table 1. General characteristics (values are number of cases (\%) unless otherwise specified)

$\begin{array}{llll} & \text { UNE } & \text { Controls } & \mathbf{p} \\ \text { Patients } & 137 & 55 & - \\ \text { Male / female patients } & 75 / 62 & 15 / 40 & 0,001 \\ \text { Mean age at onset in years (range) } & 54(25-84) & 47(19-74) & 0,0024 \\ \text { Affected side: left / right / bilateral } & 78 / 38 / 21 & 21 / 20 / 14 & 0,054 \\ \text { Most severely affected side: left / right } & 92 / 45 & 28 / 27 & 0,053 \\ \text { Median duration of symptoms in months (IQR) } 3(1-6) & 5(2-12) & 0,09 \\ \begin{array}{l}\text { Symptoms } \\ \text { Paraesthesias digit 4 / } 5\end{array} & 118(86) & 54(98) & 0,027 \\ \quad \text { Numbness digit 4 / 5 } & 115(84) & 44(80) & 0,66 \\ \quad \text { Elbow pain } & 50(36) & 23(42) & 0,60 \\ \quad \text { Cramp hand muscles } & 20(15) & 5(9) & 0,43 \\ \text { Weakness / clumsiness } & 89(65) & 37(67) & 0,89 \\ \text { Sensory signs } & & & \\ \quad \text { Normal } & 34(25) & 21(15) & 0,09 \\ \quad \text { Volair ulnar area } & 101(74) & 29(53) & 0,0082 \\ \quad \text { Dorsal ulnar area } & 77(56) & 22(40) & 0,06 \\ \text { Motor signs } & & & \\ \quad \text { Normal } & 58(42) & 41(75) & 0,0001 \\ \text { Paresis FDI or ADM } & 73(53) & 14(25) & 0,0008 \\ \text { Paresis FCU of FDP } & 51(37) & 5(9) & 0,0002\end{array}$

IQR: interquartile range

FDI: first dorsal interosseus muscle

ADM: abductor digiti minimi muscle

FCU: flexor carpi ulnaris muscle

FDP: flexor digitorum 4 and 5 profundus muscle

The control group was younger and contained more women. Weakness of ulnar muscles was found more frequently in UNE patients. 


\section{Reference test}

Of 137 patients with UNE, 75 had electrophysiological conduction abnormalities across the elbow required for UNE as well as nerve thickening during sonography, 30 had conduction abnormalities across the elbow without nerve thickening, and 32 had nerve thickening without conduction abnormalities across the elbow. However, 12 of these 32 patients had non-localizing abnormalities during electrophysiological studies (low or absent ADM / FDI CMAP, or low or absent ulnar SNAP, or abnormalities during concentric needle examination of ulnar innervated muscles). Thus 20 patients with UNE were diagnosed on the basis of nerve thickening without any electrophysiological abnormality, of whom 2 had sensory signs only, 8 sensory and motor signs and 10 symptoms but no signs.

\section{Provocative tests}

The outcome of the provocative tests is found in table 2 .

Table 2 Outcome of provocative tests in UNE and controls (in numbers of patients)

$\begin{array}{cll} & \text { UNE } & \text { Controls } \\ & \mathrm{n}=137 & \mathrm{n}=55 \\ \begin{array}{c}\text { Tinel's test } \\ \text { positive }\end{array} & 85 & 26 \\ \begin{array}{c}\text { negative } \\ \begin{array}{c}\text { Flexion compression test } \\ \text { positive }\end{array}\end{array} & 52 & 29 \\ \begin{array}{c}\text { negative } \\ \text { Palpating for nerve thickening } \\ \text { positive }\end{array} & 53 & 33 \\ \quad \text { negative } & 37 & 22 \\ \text { nerve not felt } & 93 & 46 \\ \begin{array}{c}\text { Palpating for nerve tenderness } \\ \text { positive }\end{array} & 7 & 2 \\ \text { negative } & 44 & 11 \\ & 93 & 44\end{array}$

There were no indeterminate test results except for palpation for ulnar nerve thickening because the nerve was not felt in 7 patients with UNE (5\%) and 2 controls (4\%). Compared with sonography, nerve size was correctly classified as thickened at palpation in 29 of 107 cases (21\%), and normal in 65 of 85 cases $(76 \%)$, resulting in an overall agreement between sonography and palpation of $51 \%$. Regarding the flexion compression test, patients with UNE reported tingling sensations earlier than controls, respectively after a median of 5 and 10 seconds: $p=0.0076$.

The diagnostic value of single provocative tests and combinations of tests is described in table 3. 
Table 3 Diagnostic value (and 95\% Cl) of provocative tests in UNE

\begin{tabular}{|l|c|c|c|c|c|c|}
\hline & Sensitivity & Specificity & PPV & NPV & LR + & LR - \\
\hline Tinel's test & $62 \%$ & $53 \%$ & $77 \%$ & $36 \%$ & 1,3 & 0,72 \\
& $53,7-69,7$ & $39,8-65,3$ & $67,9-83,5$ & $26,2-46,7$ & $0,98-1,72$ & $0.51-1.02$ \\
\hline $\begin{array}{l}\text { Flexion } \\
\text { compression } \\
\text { test }\end{array}$ & $61 \%$ & $40 \%$ & $72 \%$ & $29 \%$ & 1,0 & 0,99 \\
\hline $\begin{array}{l}\text { Palpating for nerve } \\
\text { thickening }\end{array}$ & $28,2-68,4$ & $28,1-53,2$ & $62,8-79,0$ & $20,0-40,0$ & $0,70-1,42$ & $0.73-1.34$ \\
\hline $\begin{array}{l}\text { Palpating for nerve } \\
\text { tenderness }\end{array}$ & $21,6-36,5$ & $75,4-93,4$ & $70,6-92,1$ & $25,8-41,3$ & $1,63-2,97$ & $0.41-1.65$ \\
\hline $\begin{array}{l}\text { One or more } \\
\text { positive } \\
\text { tests }\end{array}$ & $24,9-40,3$ & $67,6-88,5$ & $67,6-88,5$ & $24,9-40,3$ & $1,21-2,11$ & $0,49-1,46$ \\
\hline $\begin{array}{l}\text { Two or more } \\
\text { positive } \\
\text { tests }\end{array}$ & $80 \%$ & $26 \%$ & $73 \%$ & $34 \%$ & 1,1 & 0,77 \\
\hline $\begin{array}{l}\text { Three or more } \\
\text { positive } \\
\text { tests }\end{array}$ & $72,8-86,1$ & $15,8-38,3$ & $65,3-79,3$ & $21,6-49,5$ & $0,7-1,7$ & $0,5-1,1$ \\
\hline Four positive tests & $50,0-66,3$ & $41,5-67,0$ & $67,2-83,3$ & $25,3-44,9$ & $1,0-1,7$ & $0,5-1,1$ \\
\hline & $24,2-39,6$ & $71,7-91,1$ & $70,3-90,6$ & $25,6-41,0$ & $1,4-2,5$ & $0,4-1,5$ \\
\hline
\end{tabular}

PPV: positive predictive value; NPV: negative predictive value; LR +: likelihood ratio positive test; LR -: likelihood ratio negative test

None of these four tests had both good sensitivity and specificity, the diagnostic accuracy being $59 \%$ for Tinel's test, $55 \%$ for the flexion compression test, $46 \%$ for nerve tenderness and $45 \%$ for ulnar nerve thickening during palpation. Although the positive predictive value of all four tests was considerable, the negative predictive value was very low. The diagnostic value for any or more than one positive tests was also low. ROC analysis on the number of positive tests revealed an AUC of 0.59 $(95 \% \mathrm{Cl} 0.51-0.68, \mathrm{p}=0.048)$. In the group of 105 patients with an abnormal and localizing electrophysiological study sensitivities of the four provocative tests were $62 \%$ for Tinel's test, $60 \%$ for the flexion compression test, 28 for palpating for nerve thicknening and $31 \%$ for palpating for nerve tenderness. In the group of patients in whom UNE was diagnosed on the basis of nerve thickening without any electrophysiological abnormality sensitivities were $57 \%$ for Tinel's test, $64 \%$ for the flexion compression test, $25 \%$ for palpating for nerve thicknening and $32 \%$ for palpating for nerve tenderness.

We also tested specific combinations of two or more tests but this did not result in increase of sensitivity without substantial loss of specificity: e.g., having either a positive Tinel's test or a positive flexion compression test has a sensitivity of $76 \%$ but a specificity of $29 \%$ while adding nerve tenderness or nerve thickening increases specificity to respectively $84 \%$ and $89 \%$ but decreases sensitivity to $30 \%$ and $25 \%$. Limiting the time in which the flexion compression test had to be positive to $50,40,30,20,10$, or 5 seconds did not result in better specificity when combining it with a positive Tinel's test: e.g., having either a positive flexion compression test within 5 seconds or a positive Tinel's test had a sensitivity of $64 \%$ and specificity of $47 \%$. Adding the condition of an abnormal neurological exam to a positive flexion compression test or positive Tinel's test neither improved the diagnostic value: sensitivity $64 \%$ and specificity $55 \%$. The estimated probability of UNE on clinical judgement by 
the physician (probable, possible or unlikely) was associated with the final diagnosis $(p=0.001$, chi square test for trend).

The three models constructed with multiple logistic regression analysis all differed significantly. Each more comprehensive model performed significantly better. Model 2 (including motor and sensory functions obtained at routine neurological examination) was clearly better than model 1 (including only age, gender and most affected side) according to the likelihood-ratio test (14.123, $d f 1, p=0.0009)$. However, the difference between model 2 and model 3 (including all four provocative tests) was less convincing (11.715, df4, $\mathrm{p}=0.0196)$. Figure 3 shows the corresponding ROC curves for the three models. The AUC increases from $0.70(95 \% \mathrm{Cl} 0.62-0.79)$ for model 1 to $0.77(95 \% \mathrm{Cl} 0.70-0.84)$ for model 2 and $0.78(95 \% \mathrm{Cl} 0.71-0.85)$ for model 3 . This is in line with the likelihood-ratio tests: the gain is obvious for including the routine motor and sensory functions, but hardly evident for including the provocative tests.

A logistic regression model using a backward selection method resulted in a model which included age, gender, presence of paresis of ulnar innervated muscles and one provocative test (presence of ulnar thickening on palpation), but the latter did not reach significance within the model.

Plotting the post-test probability as a function of the pre-test probability for negative and positive test results (not shown) demonstrated that the gain in probability is negligible for each individual provocative test with either a positive or a negative test result. 


\section{DISCUSSION}

In this prospective study we determined the diagnostic value of provocative clinical tests for UNE in a relevant clinical spectrum of patients by making a comparison with an independently assessed reference standard comprising of electrophysiological studies and neurosonography. None of the provocative tests was found to have good diagnostic accuracy, neither single tests nor combinations of tests.

Few other investigators have studied the usefulness of provocative clinical tests in UNE and from a methodological point of view all these studies were seriously flawed. One study evaluated both Tinel's and the flexion compression test. This study contained a relatively small number of UNE patients $(n=32)$ and made a comparison with asymptomatic subjects, while it is not clear whether the index and reference tests were evaluated in a blinded fashion. Moreover, clinical severity was not reported, recruitment of patients and controls was not described, and arms were evaluated instead of patients.(5) The conclusion that sensitivities (70 and 98\%) and specificities (98 and 95\%) are reasonable to excellent should therefore be interpreted very cautiously. In another study of only 13 patients with UNE (and no controls), elbow flexion test without compression was investigated and found to be very sensitive but with the same methodological problems as described above.(6) More recently, Tinel's test and flexion compression test were evaluated in comparison with a new provocative test, the scratch collapse test, in 64 patients with UNE.(7) These authors also found a low sensitivity for the flexion compression test of $46 \%$ but with a specificity of $99 \%$, while the sensitivity of Tinel's test was only $54 \%$ with a specificity of $99 \%$. However, also in this study healthy subjects were used as controls, it is not clear if the index and reference tests were evaluated in a blinded fashion, the clinical severity was not reported, the recruitment of patients and controls was not described, and arms were evaluated instead of patients. Elbow flexion test without compression and Tinel's test were often positive in healthy subjects.(8) We found no studies on palpating for local tenderness and ulnar nerve thickening.

In our study we intentionally chose not to enroll control patients separately. To mimic the clinical situation we included consecutive patients in whom the diagnosis of UNE was included in the differential diagnosis after history taking. This resulted in a representative case-mix of patients in whom the diagnostic value of the tests is clinically relevant. The relatively small proportion of patients not suffering from UNE is probably reflecting the referral bias (high prior probability for UNE once referred to a neurologist with ulnar nerve related symptoms).

The present study has several limitations. First, although the largest study so far, the limited number of patients not suffering from UNE especially affects the precision of the specificity estimation and related concepts. Second, before the study started we discussed the potential influence of the examiner's expectation on the outcome of the provocative tests after taking a history and doing a neurological exam: e.g., the intensity of performing Tinel's test, flexion compression test and palpating for nerve tenderness may have negative or positive effects on the outcome of these tests. We could have avoided this by letting an independent neurologist perform the provocative tests but this contrasts with clinical neurological practice where history taking and testing sensory and motor functions will always be done together with other clinical tests. Third, we also considered the possible influence of one provocative test on the other: e.g., a prolonged compression during a flexion compression test might lead to a more easily elicited Tinel's sign. This effect could have been eliminated by performing only one additional test per patient (assuming that history taking and neurological exam will have no influence at all). However, this would require at least four times the number of patients while in daily practice additional tests are also combined. We tried to diminish effects between the tests by performing them in random order but positive or negative effects between the tests cannot be excluded. 
Several investigators suggested that surgery in UNE can be performed on the basis of a positive Tinel's test or flexion compression test without electrophysiological support at all or with negative electrophysiological studies. $(9,10,11)$ Our results do not support these recommendations, as the poor likelihood ratio of a positive result of one of these two tests may result in surgery of patients with conditions other than UNE. However, there is no internationally accepted gold standard for UNE and electrophysiological studies may be false negative. We added sonography to the reference tests. Although this is a relatively new test for UNE and probably not widely applied, it is an accurate test with additional value to electrophysiological tests.(2) In this study $23 \%$ of the patients were finally diagnosed with UNE by demonstrating focal ulnar nerve thickening at the elbow while more than one-third of these patients had nonlocalizing electrophysiological abnormalities. Although the use of sonography in our study resulted in the inclusion of electrophysiological negative cases of UNE, the diagnostic performance of provocative tests was still poor. The results of the provocative tests in electrophysiological negative cases were in line with the results in the total group and with the group with an abnormal and localizing electrophysiological test. Therefore the introduction of sonography to the reference test did not lead to an important bias on the results of the provocative tests and indicate that provocative tests cannot be used as an alternative test to make a diagnosis of UNE more likely when electrophysiological tests are negative.

We conclude that the diagnostic value of provocative tests in UNE is poor and should not be recommended for clinical decision making.

\section{Competing interests}

None of the authors has competing interests to declare.

\section{Titles and legends to the figures}

\section{Figure 1: Sonogram}

Longitudinal sonogram of an enlarged ulnar nerve (indicated by black arrows) at the level of the medial epicondyle. The ulnar nerve diameter within the echogenic rim surrounding the nerve is $3.6 \mathrm{~mm}$ (white dotted line).

Figure 2: Flow diagram

FC test, flexion compression test; UN, ulnar nerve; ulnar nerve not felt in 6 cases $^{\star}$ and 3 cases $^{\star *}$

Figure 3: ROC curves of the three multiple logistic regression models

Areas under the curve (AUCs): model 1: 0,70; model 2: 0,77; model 3: 0,78. AUCs are standardized measures of the diagnostic performance of a diagnostic test or procedure. Each subsequent logistic model was compared to its predecessor using likelihood ratio tests (see text). 


\section{REFERENCES}

1. Stewart JD. The variable clinical manifestations of ulnar neuropathies at the elbow. $J$ Neurol Neurosurg Psychiatry 1987;50:252-58.

2. Beekman R, Schoemaker MC, van der Plas JP, et al. The diagnostic value of high-resolution sonography in ulnar neuropathy at the elbow. Neurology 2004;62:767-73.

3. Stewart JD. Ulnar neuropathies: where, why, and what to do? Practical Neurology 2006;6;21829.

4. Krom de MC, Knipschild PG, Kester AD, et al. Efficacy of provocative tests for diagnosis of carpal tunnel syndrome. Lancet 1990;335:393-95.

5. Novak CB, Lee GW, Mackinnon SE, et al. Provocative testing for cubital tunnel syndrome. $J$ Hand Surg 1994;19:817-20.

6. Buehler MJ, Thayer D. The elbow flexion test. A clinical test for the cubital tunnel syndrome. Clin Orthop 1988;233:213-16.

7. Cheng CJ, Mackinnon-Patterson B, Beck JL, et al. Scratch collapse test for evaluation of carpal and cubital tunnel syndrome. J Hand Surg [Am] 2008;33:1518-24.

8. Kuschner SH, Ebramzadeh E, Mitchell S. Evaluation of elbow flexion and Tinel tests for cubital tunnel syndrome in asymptomatic individuals. Orthopedics 2006;29:305-8.

9. Tomaino MM, Brach PJ, Vansickle DP. The rationale for and efficacy of surgical intervention for electrodiagnostic-negative cubital tunnel syndrome. J Hand Surg 2001;26A:1077-81.

10. Greenwald D, Moffitt M, Cooper MD. Effective surgical treatment of cubital tunnel syndrome based on provocative clinical testing without electrodiagnostics. Plast Reconstr Surg 1999;104:215-18.

11. Greenwald D, Blum LC, Adams D, et al. Effective surgical treatment of cubital tunnel syndrome based on provocative clinical testing without electrodiagnostics. Plast Reconstr Surg 2006;117:87e-91e. 


\section{Statement}

The Corresponding Author has the right to grant on behalf of all authors and does grant on behalf of all authors, an exclusive licence (or non exclusive for government employees) on a worldwide basis to the BMJ Publishing Group Ltd and its Licensees to permit this article (if accepted) to be published in the Journal of Neurology, Neurosurgery \& Psychiatry editions and any other BMJPGL products to exploit all subsidiary rights, as set out in their licence (http://jnnp .bmjjournals.com/ifora/licence.pdf)." 


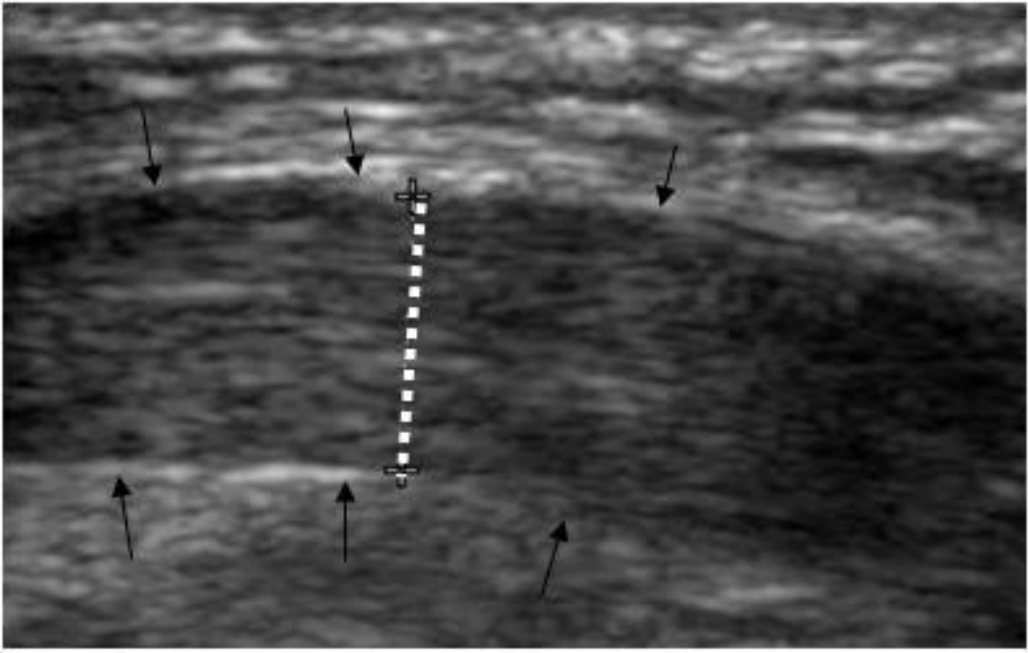




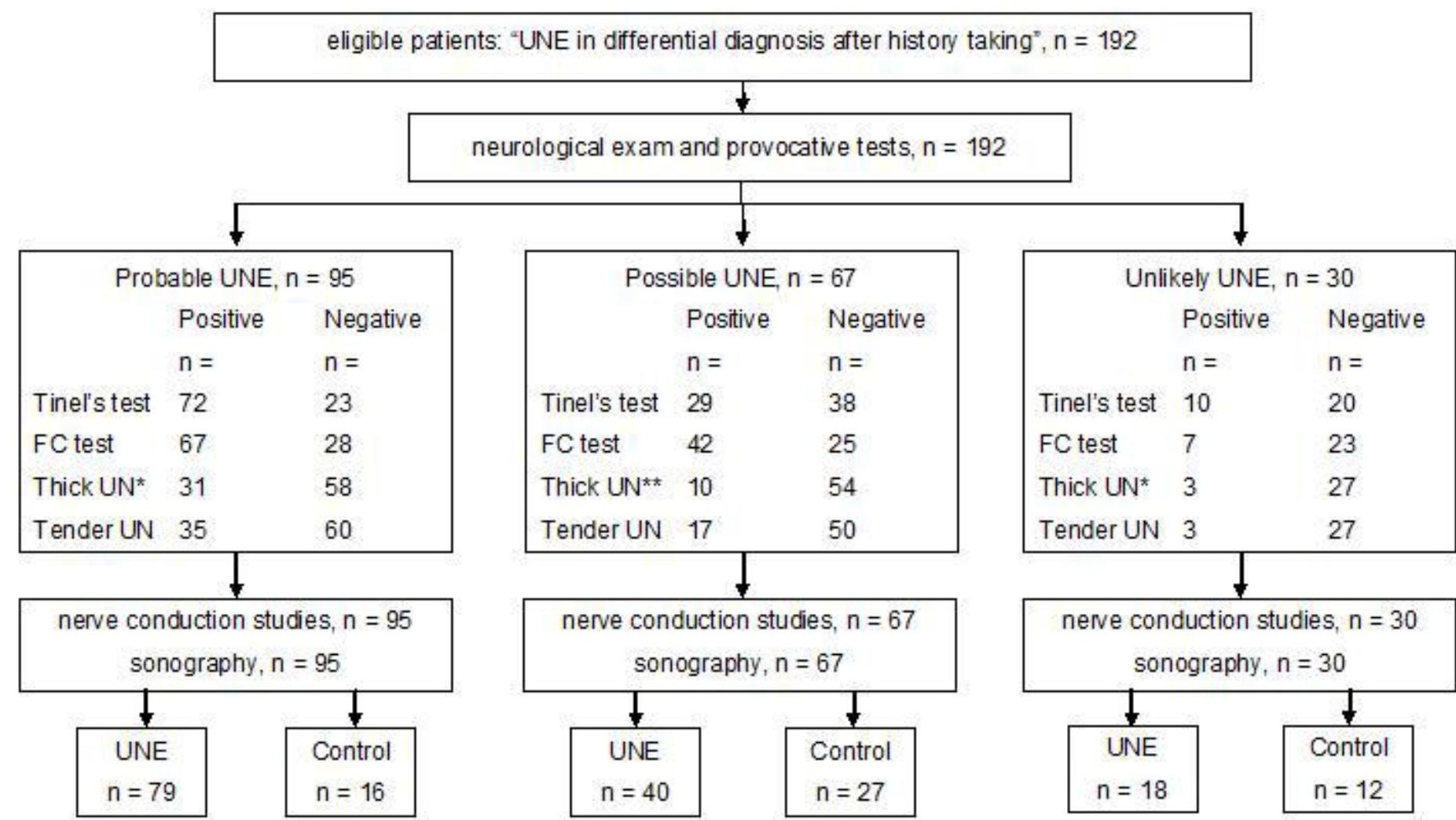




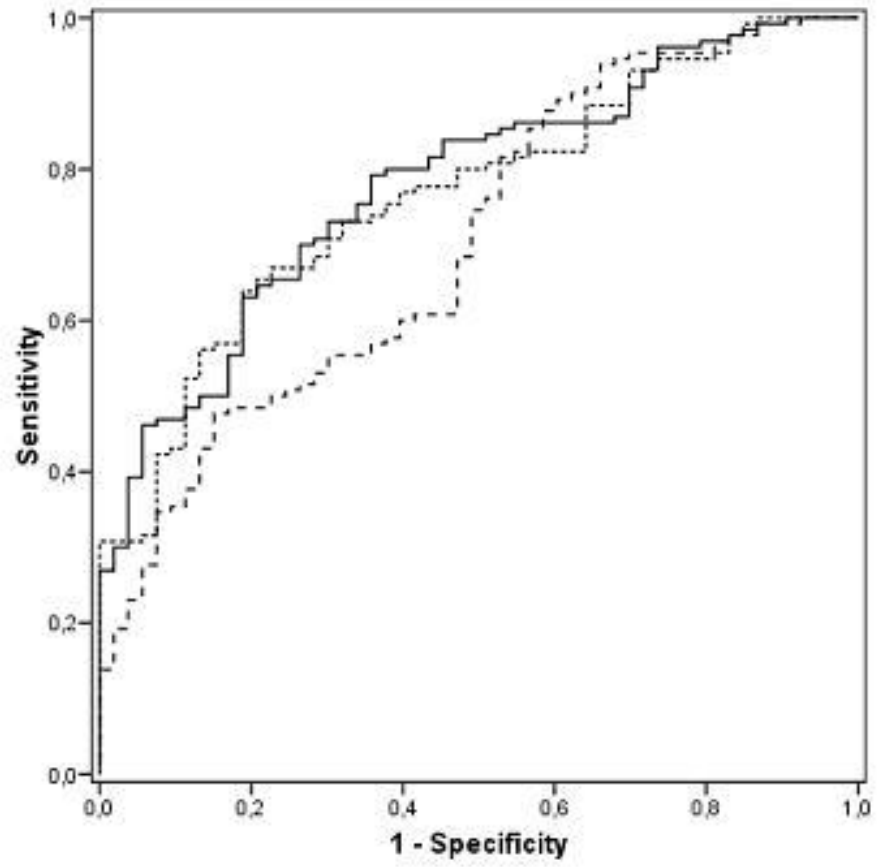

- - Model 1

..... Modiel 2

- Model 3 


\section{INNP The diagnostic value of provocative clinical tests in ulnar neuropathy at the elbow is marginal}

Roy Beekman, Tobien Schreuder, Stan Rozeman, et al.

J Neurol Neurosurg Psychiatry published online June 23, 2009

doi: 10.1136/jnnp.2009.180844

Updated information and services can be found at:

http://jnnp.bmj.com/content/early/2009/06/23/jnnp.2009.180844

These include:

$\mathbf{P}<\mathbf{P} \quad$ Published online June 23, 2009 in advance of the print journal.

Email alerting Receive free email alerts when new articles cite this article. Sign up in the service box at the top right corner of the online article.

Notes

Advance online articles have been peer reviewed and accepted for publication but have not yet appeared in the paper journal (edited, typeset versions may be posted when available prior to final publication). Advance online articles are citable and establish publication priority; they are indexed by PubMed from initial publication. Citations to Advance online articles must include the digital object identifier (DOls) and date of initial publication.

To request permissions go to:

http://group.bmj.com/group/rights-licensing/permissions

To order reprints go to:

http://journals.bmj.com/cgi/reprintform

To subscribe to BMJ go to:

http://group.bmj.com/subscribe/ 